\title{
INFLUENCIA DA INGESTÃO ALIMENTAR SOBRE O HEMOGRAMA
}

\author{
Dorisdaia Carvalho de Humerez* \\ Else Duarte* \\ Emília Campos** \\ Márcia Caron Ruffino* \\ Tokico Murakawa Moriya***
}

\begin{tabular}{l|l|}
\cline { 2 - 2 } & $\mathrm{RBEn} / 07$ \\
\hline
\end{tabular}

HUMEREZ, D.C., colaboradores - Influência da ingestão alimentar sobre o hemograma.

Rev. Bras. Enf.; RJ, $28: 54-59,1975$.

Uma das análises necessárias à classe médica e que pode oferecer importantes indicações no que se refere ao diagnóstico e terapêutica é o hemograma (1).

Os laboratórios fazem geralmente outros tipos de exames e, como para muitos deles é exigido que o paciente esteja em jejum, ficou então, estabelecido como norma geral que o hemograma deva também ser feito nestas condições (5).

VIEIRA ROMEIRO (10), LIMA e cols. (6) recomendam que o sangue para exame hematológico deve ser colhido em jejum de várias horas. Entretanto, para JANNINI \& JANNINI, F. ${ }^{\circ}$ (5) o hemograma pode ser feito a qualquer hora, antes ou depois da alimentação.

Em alguns casos, o exame hematológico é pedido com a finalidade de auxílio diagnóstico quando o paciente está febril. Assim, a urgência do exame, a tensão dos familiares, não permitem que se espere até o dia seguinte para a colheita do material em jejum (5).

Acresce ainda o problema dos pacientes de ambulatório, residentes em outras cidades, que viajam para fazer o exame e, às vezes, pela orientação inadequada alimentam-se ficando, assim, impedidos de realizar o exame, causando-lhes sérios transtornos de ordem econômica. emocional e retardo no início do tratamento. Quando os pacientes internados se alimentam, a colheita do material fica também para o dia seguinte, salvo resolução médica em contrário.

Como se tem verificado, quando o organismo é acometido de processo febril, se o sangue for colhido no dia seguinte para esperar que o paciente esteja em jejum, o quadro hematológico deste dia pode mascarar o processo anterior e, nestas condições, o hemograma será a característica do quadro atual e não a do que se desejava analisar (5).

* Auxiliar de Ensino do Departamento de Enfermagem Geral e Especializada, Escola de Enfermagem de Ribeirão Preto, Universidade de São Paulo.

** Auxiliar de Ensino Voluntário do Departamento de Enfermagem Geral e Especializada, Escola de Enfermagem de Ribeirão Preto, Universidade de São Paulo.

*** Professor-Colaborador do Departamento de Enfermagem Geral e Especializada, Escola de Enfermagem de Ribeirão Preto, Universidade de São Paulo. 
HUMEREZ, D.C., colaboradores - Influência da ingestão alimentar sobre o hemograma. Rev. Bras. Enf.; RJ, $28: 54-59,1975$.

Sabe-se que, em circunstâncias fisiológicas, pode haver desvios da fórmula leucocitária. E comum a leucocitose fisiológica do recém-nascido, podendo ainda ser observada no período menstrual, na gravidez, no parto e exercícios musculares exaustivos; de maneira análoga pode surgir leucocitose como resultado da secreção de adrenalina nos casos de "stress", medo, dor e anóxia $(8,3)$.

Com relação a alimentação, parece ainda haver divergência de opiniões se o sangue para hemograma deve ou não ser colhido em jejum e se as alterações ocorridas tem ou não siginificado.

Considerando a importância do exposto, propusemo-nos a estudar a influência da ingestão alimentar sobre o hemograma.

\section{MATERIAL E MÉTODOS}

Selecionou-se como população de estudo, universitárias de Ribeirão Preto, como idade entre 19 e 29 anos.

Foram incluídas no estudo, 20 voluntárias sem queixa de moléstia, com sinais vitais dentro dos limites de normalidade e que não estivessem recebendo tratamento medicamento. Chamamos de limites de normalidade a temperatura entre $36,2 .^{\circ} \mathrm{C}$ e $36,9 .{ }^{\circ} \mathrm{C}$; a freqüência cardíaca entre 62 e 97 batimentos $/ \mathrm{min}$; a pressão arterial sistólica entre 135,84 e $99,09 \mathrm{~mm} \mathrm{Hg}$ a diastólica entre 87,03 e $57,12 \mathrm{~mm} \mathrm{Hg}(4,7,11)$.

Os indivíduos foram divididos casualmente, através de sorteio, em dois grupos: experimental e controle.

Em ambos os grupos foi colhida uma primeira amostra de sangue pela manhã, através de punção venosa, estando os indivíduos em jejum por um período mínimo de 8 horas, e em repouso de pelo menos 10 minutos.

No grupo experimental, após a primeira colheita, cada elemento recebeu o desjejum que constou de café com leite adoçado $(200 \mathrm{ml})$, pão $(50 \mathrm{~g})$ com manteiga $(10 \mathrm{~g})$. Após 60 minutos do desjejum, colheu-se uma segunda amostra de sangue em idênticas condições da primeira amostra.

No grupo controle as participantes não receberam alimentação e foi colhida uma segunda amostra simultâneamente ao seu par do grupo experimental.

Todas as amostras de sangue variando de 4,0 a $4,5 \mathrm{ml}$ foram colocadas em frascos que comportam $10 \mathrm{ml}$ (padronizados pelo Hospital das Clínicas de Ribeirão Preto, USP) que continham como anticoagulante 50 de EDTA (Na+10\%) Após serem codificados (código numérico), os frascos foram encaminhados ao laboratório de hematologia para contagem de eritrócitos e leucócitos, e dosagem de hemoglobina, onde utiliza-se o aparelho Coulter-Counter modelo F.N. para realização de exames deste tipo (9).

Com interesse em comparar resultados das variáveis entre grupo experimental e grupo controle e, sabendo-se que existe uma variabilidade biológico inerente a esta medida, acrescido do erro de técnica, efetuamos numa primeira etapa a medida desta variabilidade e do erro. Isto foi feito da seguinte maneira: foi colhido sangue de quatro indivíduos. De cada indivíduo colheu-se $18 \mathrm{ml}$ de sangue que foi distribuído em quatro frascos idênticos contendo o mesmo anticoagulante citado anteriormente, codificados (código numérico) e enviados ao laboratório para contagem dos eritrócitos e leucócitos, e dosagem de hemoglobina.

\section{RESULTADOS E DISCUSSÃO}

A contagem dos G.V. (glóbulos vermelhos), G.B. (glóbulos brancos) e dosagem da $\mathrm{Hb}$ (hemoglobina) do grupo experimental e grupo controle, são apresentados na tabela $I$. 


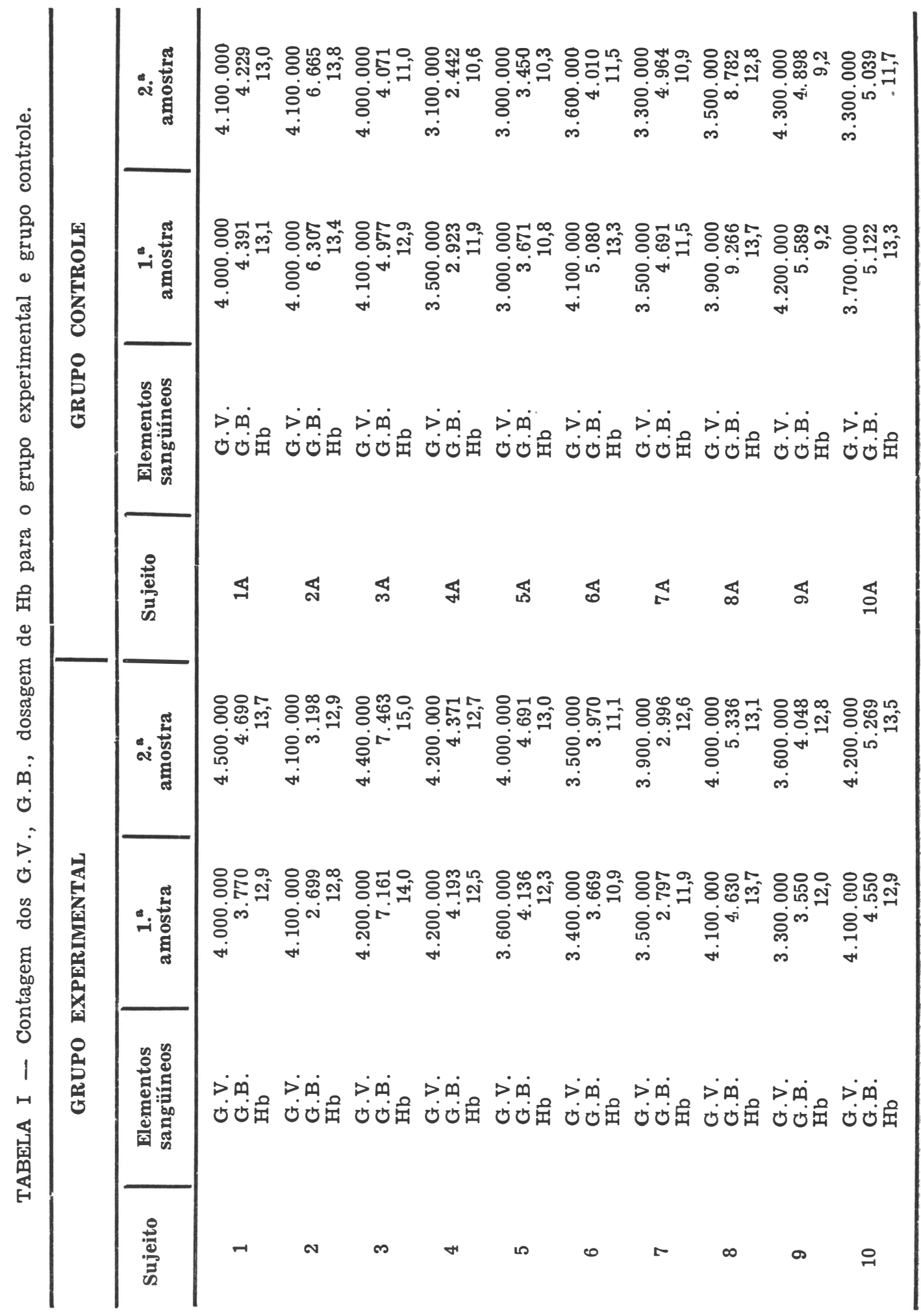


HUMEREZ, D.C., colaboradores - Influência da ingestão alimentar sobre o hemograma.

Rev. Bras. Enf.; RJ, 28 : 54-59, 1975.

A tabela II mostra as diferenças entre as variáveis obtidas na $1 .^{\text {a }}$ amostra e $2 .^{a}$ amostra e ainda as medidas de posição e variabilidade. Para estes da- dos, ao se efetuar o teste de duas médias para populações correlatas encontrou-se valores de $\underline{\mathbf{t}}$ que são assinalados na tabela II.

TABELA II - Diferença entre as variáveis obtidas na primeira e segunda amostra para grupo experimental e para grupo controle.

\begin{tabular}{|c|c|c|c|c|c|c|c|}
\hline \multicolumn{4}{|c|}{ GRUPO EXPERIMENTAL } & \multicolumn{4}{|c|}{ GRUPO CONTROLE } \\
\hline Sujeito & $\mathbf{G} \cdot \mathbf{V}$. & G.B. & $\mathbf{H b}$ & Sujeito & $\mathbf{G} \cdot \mathbf{V}$ & G.B. & $\mathbf{H b}$ \\
\hline 1 & 500.000 & 920 & $-0,8$ & $1 \mathrm{~A}$ & 100.000 & -162 & $-0,1$ \\
\hline 2 & 0 & 499 & $-0,1$ & $2 A$ & 100.000 & 358 & 0,4 \\
\hline 3 & 200.000 & 302 & $-1,0$ & $3 A$ & -100.000 & -906 & $-1,1$ \\
\hline 4 & 0 & 178 & $-0,2$ & $4 \mathrm{~A}$ & -400.000 & -481 & $-1,3$ \\
\hline 5 & 400.000 & $5 \cdot 55$ & 0,7 & $5 \mathrm{~A}$ & 0 & -221 & $-0,5$ \\
\hline 6 & 100.000 & 301 & 0,2 & $6 \mathrm{~A}$ & -500.000 & -1.070 & $-2,2$ \\
\hline 7 & 400.000 & 199 & 0,7 & $7 \mathrm{~A}$ & -200.000 & 273 & $-0,6$ \\
\hline 8 & -100.000 & 706 & $-0,6$ & $8 \mathrm{~A}$ & -400.000 & -484 & $-1,1$ \\
\hline 9 & 300.000 & 498 & 0,8 & $9 \mathrm{~A}$ & 100.000 & -691 & 0 \\
\hline 10 & 100.000 & 719 & 0,6 & $10 \mathrm{~A}$ & -400.000 & -83 & $-1,6$ \\
\hline AT & 600.000 & 742 & 1,8 & $\mathrm{AV}$ & 600.000 & 1.428 & 2,6 \\
\hline$\overline{\mathbf{x}}$ & 190.000 & 487,70 & 0,03 & $\bar{x}$ & -170.000 & $-346,70$ & $-8,81$ \\
\hline $\mathrm{s}$ & 19.972 & 245,27 & 0,67 & s & 240.601 & 470,40 & 0,84 \\
\hline$\underline{t}$ & 3,00 & 6,28 & 0,14 & $\underline{t}$ & $-2,22$ & $-2,33$ & $-3,00$ \\
\hline \\
\hline \multicolumn{8}{|c|}{$\begin{array}{l}\overline{\mathbf{x}} \text { - Média aritmética } \\
\mathrm{s} \quad \text { - Desvio padrão }\end{array}$} \\
\hline & teste de $2 \mathrm{~m}$ & dias para & pulac & orrela & & & \\
\hline
\end{tabular}

Da observação da tabela II, no teste de duas mécias para populações correlatas $(\propto=0,05)$ vemos que, para 0 grupo experimental, a alteração é significante para G.V. e G.B. não sendo, porém, significante para. Hb. Para o grupo controle a variação é significante para os G.V., G.B. e Hb.
Os resultados do estudo para verificar a variabilidade biológica inerente a esta medida acrescida do erro de técnica, na contagem de eritrócitos, leucócitos e dosagem de hemoglobina são mostrados na tabela III. 


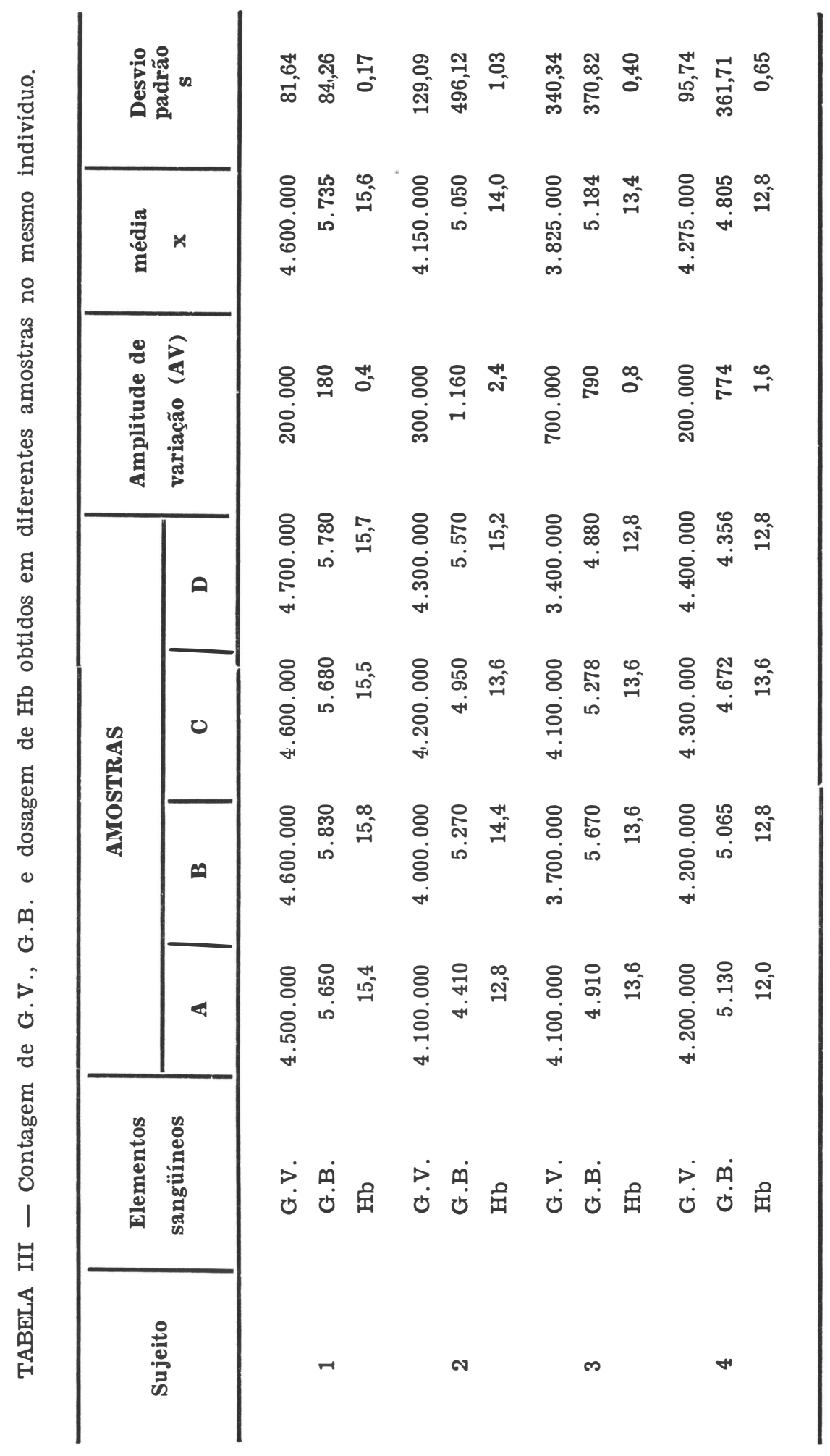


HUMEREZ, D.C., colaboradores - Influência da ingestão alimentar sobre o hemograma. Rev. Bras. Enf.; RJ, $28: 54-59,1975$.

As alterações que ocorrem nos dois grupos, conforme tabela II, situam-se dentro da variabilidade da medida inerente à técnica (tabela III); embora tenha ocorrido elevação dos elementos sangüíneos no G.E., que teve a alimentação como uma variável a mais e, no G.C. em que esses elementos diminuiram estando os indivíduos em jejum sentimos necessidade de estudos posteriores para estabelecer relação entre essas variações.

\section{REFERENCIAS}

1. BARBOSA, N.C. - Interpretação do hemograma. An. Brasil. Ginec. 55: 133-138, 1963

2. BETHELL, F. et al. - Quantitative and qualitative variations in normal leucocytes. Physiological Rev., 23: 279-301, 1943.

3. DEPLA, M. et FUNEL, P. - Variation spontanée de l'hemograme moyen d'une population de travailleurs. Rev. Franc. Études Clin. et Biol., 7: 752-754, 1962.

4. HOUSSAY, B.A. et al. - Temperatura corporal y su regulacion. In: Fisiologia humana. 4. ${ }^{\mathrm{a}}$ ed. Buenos Aires, El Teneo, cap. 52, 1972.

5. JANNINI, P. JANNINI FILHO, P. Colheita do material e técnica de exame. In:- Interpretação clínica do hemograma. 7.a ed., São Paulo, Gráfica São José, cap. 2, 1973.

6. LIMA, A.O. et al. - Métodos de laboratório aplicados à clínica. $4 .^{\mathrm{a}}$
Nas condições em que se apresentam nossos resultados e em que o experimento foi realizado, chegamos a admitir que, na colheita de sangue para hemograma, a alimentação não provoca alteração dos elementos sangüíneos.

Estendemos nossos agradecimentos ao Prof. Dr. Antonio Ruffino Netto, Assistente Doutor do Departamento de $\mathrm{Me}$ dicina Social da Faculdade de Medicina de Ribeirão Preto, USP, pelas sugestões apresentadas.

\section{BIGLIOGRAFICAS}

ed., Rio de Janeiro, Ed. Guanabara Koogan, 1969.

7. MACHADO, M.H. - Freqüência cardíaca, freqüência respiratória em pessoas do grupo etário $14-19$ anos. Ribeirão $P$ reto, 1972, Tese de doutoramento, Escola de Enmagem de Ribeirão Preto, USP, (Mimeogr.).

8. NERY, A.L.B. et al - Hemograma: série branca. In:- Laboratório para o clínico. Rio de Janeiro, Livraria Atheneu, cap. 6, 1973.

9. PLATT, W.L. - Color atlas and textbook of hematology. Priladelphia Toronto J.B. Lippincott, 1969.

10. VIEIRA ROMEIRO - Exame do sangue. In:- Semiologia médica. 11. ${ }^{\text {a }}$ ed., Rio de Janeiro, Ed. Guanabara Koogan, cap. 10, 1968.

11. VINHA, V.H.P. - Estudo da yressão arterial em policiais militares do grupo etário $20-50$ anos. Ribeirão Preto, USP, (mimeograf.). 